\title{
ADAPTIVE FUZZY CONTROL FOR A CLASS OF CONSTRAINED NONLINEAR SYSTEMS WITH APPLICATION TO A SURFACE VESSEL
}

\author{
Mehrnush Sadat Jamalzade, Hamid Reza Koofigar, Mohammad Ataei \\ Department of Electrical Engineering, University of Isfahan, Isfahan, Iran \\ e-mail:koofigar@eng.ui.ac.ir
}

\begin{abstract}
In this paper, adaptive control for a class of uncertain nonlinear systems with input constraints is addressed. The main goal is to achieve a self-regulator PID controller whose coefficients are adjusted by using some adaptive fuzzy rules. The constraints on the control signal are taken into account as a saturation operator. The stability of the closed-loop system is analytically proved by using the Lyapunov stability theorem. The proposed method is then applied to a surface vessel with uncertain dynamic equations. The simulation results show the effectiveness of the proposed control strategy.
\end{abstract}

Keywords: self-regulator, fuzzy PID controller, constraint nonlinear systems, uncertainty, fuzzy estimation

\section{Introduction}

Dealing with the control problem of uncertain systems, various algorithms have been developed ensuring the robust stability and performance (Petersen and Tempo, 2014). Robust adaptive control has been formulated for a class of uncertain nonlinear systems by output feedback control (Xu and Huang, 2010; Lee, 2011). For nonlinear systems in the strict-feedback form with unknown static parameters, a robust adaptive control law was designed by Montaseri and Mohammad (2012), which guarantees the asymptotic output tracking despite matched and unmatched uncertainties. The neural-network-based robust control design, via an adaptive dynamic programming approach, was investigated in (Wang et al., 2014) to obtain the optimal performance under a specified cost function. Some applications have been also introduced in the literature, in the presence of time-varying uncertainties and disturbances (Koofigar and Amelian, 2013). Nevertheless, taking the input constraint in the controller design procedure is still highly desired.

In the last decade, a considerable attention has been paid to robust control of nonlinear systems with input constraints (Chen et al., 2010, 2014; Lu and Yao, 2014). In such cases, fuzzy logic and neural networks may be some alternative solutions. A direct adaptive fuzzy control approach has been presented for uncertain nonlinear systems in the presence of input saturation by incorporating a new auxiliary design system and Nussbaum gain functions (Li et al., 2013). The problem of adaptive fuzzy tracking control for a class of pure-feedback nonlinear systems with input saturation was studied by Wang et al., (2013a,b). Muñoz and Marquardt (2013) focused on the control design for input-output feedback linearizable nonlinear systems with bounded inputs and state constraints. An indirect adaptive fuzzy control scheme was developed for a wider class of nonlinear systems with the input constraint and unknown control direction by Wuxi et al. (2013) and Yongming et al. (2014). To this end, a barrier Lyapunov function and an auxiliary design system were employed.

From an application viewpoint, the surface vessels with uncertain nonlinear dynamics may be adopted to demonstrate the effectiveness of various control schemes. Nonlinear strategies 
(Daly et al., 2012), adaptive control (Fang et al., 2004), and neural networks (Dai et al., 2015) are samples of control algorithms in the previous investigations. Removing some drawbacks of such works, adaptive intelligent methods as adaptive neural networks, were presented by Li et al. (2015). In this study, an adaptive fuzzy algorithm is proposed to achieve the advantages of both intelligent and adaptive mechanisms for ensuring the robustness properties and taking the constraints into account.

Briefly discussing, there may exist some main restrictions in the previous investigations as, i) the fuzzy rules have been designed off-line and the stability and performance may be lost with changing the circumstances, ii) the stability analysis has not been presented in an analytical form, and iii) to ensure the stability of the closed-loop system, the initial value for the controller parameters must be set. To eliminate the aforementioned limitations, a self-regulator fuzzy PID controller is proposed in this paper, which guarantees the robustness properties against the system uncertainties and external disturbances.

This paper is organized as follows. In Section 2, the problem formulation and the constraints on input signal are introduced. In Section 3, an adaptive fuzzy controller is designed for a class of uncertain nonlinear systems with constrained input and the stability proof is given. The proposed method is applied to a surface vessel in Section 4 and the simulation results are presented. The concluding remarks are finally given in Section 5.

\section{Problem formulation}

Consider a class of nonlinear systems, represented by the state-space description

$$
\begin{aligned}
& \dot{\mathbf{X}}_{1}=\mathbf{X}_{2} \\
& \dot{\mathbf{X}}_{2}=\mathbf{X}_{3} \\
& \vdots \\
& \dot{\mathbf{X}}_{n-1}=\mathbf{X}_{n} \\
& \dot{\mathbf{X}}_{n}=\mathbf{F}\left(\mathbf{X}_{1}, \mathbf{X}_{2}, \ldots, \mathbf{X}_{n}\right)+\mathbf{G}\left(\mathbf{X}_{1}, \mathbf{X}_{2}, \ldots, \mathbf{X}_{n}\right) \mathbf{p}(\mathbf{u})+\mathbf{d}(t) \\
& \mathbf{Y}=\mathbf{X}_{1}
\end{aligned}
$$

where $\mathbf{X} \in \mathbb{R}^{n \times m}$ denotes the vector of state variables, $d(t)$ represents the external disturbance, and $\mathbf{p}(\mathbf{u}) \in \mathbb{R}^{m}$ is the vector of constrained inputs.

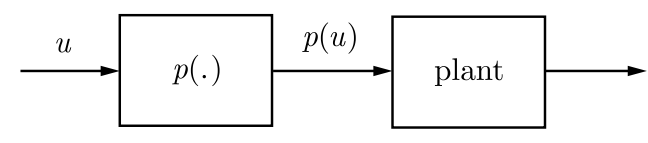

Fig. 1. Block diagram of $p(\mathbf{u})$

As schematically depicted in Fig. 1, the nonlinear operator $p(\mathbf{u})$ acts as a saturation constraint as

$$
p\left(u_{i}\right)=\left\{\begin{array}{ll}
\alpha u_{u} & \text { for } u_{i} \geqslant u_{u} \\
\alpha u_{i} & \text { for } \quad u_{l} \leqslant u_{i} \leqslant u_{u} \\
\alpha u_{l} & \text { for } \quad u_{i} \leqslant u_{l}
\end{array} \quad i=1,2, \ldots, m\right.
$$

where $u_{u}, u_{l}$ and $\alpha$ denote the parameters of saturation operator.

The saturation operator $p\left(u_{i}\right)$ is described here as

$$
p\left(u_{i}\right)=a\left(u_{i}\right) u_{i}+b\left(u_{i}\right)
$$


where $a\left(u_{i}\right)$ and $b\left(u_{i}\right)$ are given by

$$
\begin{aligned}
& a\left(u_{i}\right)= \begin{cases}0 & \text { for } \quad u_{i} \geqslant u_{u} \\
\alpha & \text { for } \quad u_{l} \leqslant u_{i} \leqslant u_{u} \\
0 & \text { for } \quad u_{i} \leqslant u_{l}\end{cases} \\
& b\left(u_{i}\right)=\left\{\begin{array}{lll}
\alpha u_{u} & \text { for } \quad u_{i} \geqslant u_{u} \\
0 & \text { for } \quad u_{l} \leqslant u_{i} \leqslant u_{u} \\
\alpha u_{l} & \text { for } \quad u_{i} \leqslant u_{l}
\end{array}\right.
\end{aligned}
$$

Incorporating description (2.3) into (2.1), yields

$\dot{\mathbf{X}}_{1}=\mathbf{X}_{2}$

$\dot{\mathbf{X}}_{2}=\mathbf{X}_{3}$

$\dot{\mathbf{X}}_{n-1}=\mathbf{X}_{n}$

$\dot{\mathbf{X}}_{n}=\mathbf{F}+\mathbf{G b}(\mathbf{u})+\mathbf{G a}(\mathbf{u}) \mathbf{u}+\mathbf{d}(t)=\mathbf{F}+\mathbf{G} \mathbf{b}(\mathbf{u})+\widehat{\mathbf{G}}_{u} \mathbf{u}+\mathbf{d}(t)$

$\mathbf{Y}=\mathbf{X}_{1}$

Remark 1. The only information about the system model is that the invertible matrix $\widehat{\mathbf{G}}_{u}(\cdot)$, as an estimate of $\mathbf{G}_{\mathbf{u}}(\cdot)=\mathbf{G}(\cdot) a(u)$, is available, see Mclain et al. (1999).

The control objective is to design the control input $\mathrm{u}$ such that $\mathbf{Y}$ tracks the smooth reference trajectory $\mathbf{Y}_{d}$. Define the tracking error vector $\mathbf{E}=\left[e_{1}, \ldots, e_{m}\right]^{\mathrm{T}}$ as

$$
\mathbf{E}=\mathbf{X}_{1}-\mathbf{Y}_{d}=\mathbf{Y}-\mathbf{Y}_{d}
$$

A PID control structure is adapted here as

$$
u_{i}=k_{P i} e_{i}+k_{I i} \int_{0}^{t} e_{i}(\tau) d \tau+k_{D i} \frac{d e_{i}}{d t} \quad i=1,2, \ldots, m
$$

where $e_{i}$ is the $i$-th component of the error vector $\mathbf{E}$, and $k_{P i}, k_{I i}$ and $k_{D i}$ denote respectively the proportional, integral and derivative coefficients.

\section{Adaptive fuzzy controller design}

\subsection{Fuzzy estimation}

In this Section, the $l$-th fuzzy rule of the fuzzy controller for estimating the unknown function $H(x)$ is formed by (Shaocheng et al., 2000)

$$
R^{l}: \quad \text { if } x_{1}=A_{1}^{l} \wedge x_{2}=A_{2}^{l} \quad \rightarrow \quad H(x)=\theta_{l}
$$

where $\mathbf{x}=\left[x_{1}, x_{2}\right]^{\mathrm{T}}$ denotes the input vector, $A_{i}^{l}$ is the membership function of each input. The fuzzy model for describing $H(\mathbf{x})$ is Mamdani, and the output of the fuzzy system can be obtained by

$$
H(\mathbf{x})=\frac{\sum_{l=1}^{N} \theta_{l} \prod_{i=1}^{2} \mu_{A_{i}^{l}}\left(x_{i}\right)}{\sum_{l=1}^{N} \prod_{i=1}^{2} \mu_{A_{i}^{l}}\left(x_{i}\right)}
$$


where $u_{F_{i}^{l}}$ is the fuzzy membership function and $N$ is the number of rules. Now, form the unknown function as

$$
H(\mathbf{x})=\mathbf{\Phi}(\mathbf{x})^{\mathrm{T}} \boldsymbol{\theta}
$$

where

$$
\begin{aligned}
& \boldsymbol{\Phi}(\mathbf{x})=\left[\phi_{1}(\mathbf{x}), \phi_{2}(\mathbf{x}), \ldots, \phi_{N}(\mathbf{x})\right]^{\mathrm{T}} \quad \phi_{l}(\mathbf{x})=\frac{\prod_{i=1}^{2} \mu_{A_{i}^{l}}\left(x_{i}\right)}{\sum_{l=1}^{N} \prod_{i=1}^{2} \mu_{A_{i}^{l}}\left(x_{i}\right)} \\
& \boldsymbol{\theta}=\left[\theta_{1}, \theta_{2}, \ldots, \theta_{N}\right]^{\mathrm{T}}
\end{aligned}
$$

\subsection{Controller design}

To facilitate the designing procedure, new state variables are defined here as

$$
\begin{aligned}
& \mathbf{Z}_{1}=\int_{0}^{t} \mathbf{E}(\tau) d \tau \\
& \mathbf{Z}_{2}=\mathbf{E} \\
& \mathbf{Z}_{3}=\frac{d \mathbf{E}}{d t} \\
& \vdots \\
& \mathbf{Z}_{n+1}=\frac{d^{n-1} \mathbf{E}}{d t^{n-1}}
\end{aligned}
$$

by which the dynamic equations (2.1) may be rewritten as

$$
\begin{aligned}
& \dot{\mathbf{Z}}_{1}=\mathbf{Z}_{2} \\
& \dot{\mathbf{Z}}_{2}=\mathbf{Z}_{3} \\
& \vdots \\
& \dot{\mathbf{Z}}_{n}=\mathbf{Z}_{n+1} \\
& \dot{\mathbf{Z}}_{n+1}=\mathbf{F}_{t}+\widehat{\mathbf{G}}_{u} \mathbf{u}
\end{aligned}
$$

where

$$
\mathbf{F}_{t}=\mathbf{F}-\frac{d^{n} \mathbf{Y}_{d}}{d t^{n}}+\mathbf{G} \mathbf{b}(\mathbf{u})+\left(\mathbf{G}_{u}-\widehat{\mathbf{G}}_{u}\right) \mathbf{u}+\mathbf{d}(t)
$$

Hence, input signal (2.7) may be given by

$$
\mathbf{u}=\mathbf{K}_{I} \mathbf{Z}_{1}+\mathbf{K}_{P} \mathbf{Z}_{2}+\mathbf{K}_{D} \mathbf{Z}_{3}
$$

where

$$
\begin{aligned}
& \mathbf{K}_{P}=\operatorname{diag}\left[\begin{array}{llll}
k_{P 1} & k_{p 2} & \cdots & k_{p m}
\end{array}\right] \\
& \mathbf{K}_{I}=\operatorname{diag}\left[\begin{array}{llll}
k_{I 1} & k_{I 2} & \cdots & k_{I m}
\end{array}\right] \\
& \mathbf{K}_{D}=\operatorname{diag}\left[\begin{array}{llll}
k_{D 1} & k_{D 2} & \cdots & k_{D m}
\end{array}\right]
\end{aligned}
$$

Now, define an ideal control signal $\mathbf{u}^{*}$ as

$$
\mathbf{u}^{*}=\boldsymbol{\Phi}_{T}^{\mathrm{T}} \boldsymbol{\Theta}^{*}=\widehat{\mathbf{G}}_{u}^{-1}\left(-\mathbf{F}_{t}-\mathbf{K}_{1} \mathbf{Z}_{1}-\mathbf{K}_{2} \mathbf{Z}_{2}-\ldots-\mathbf{K}_{n+1} \mathbf{Z}_{n+1}\right)
$$

where $\mathbf{u}^{*}$ is obtained from the feedback linearization of system (3.6). 
Remark 2. $\mathbf{K}_{i}, \quad i=1, \ldots, n+1$, is chosen such that

$$
\mathbf{A}_{c l}=\left[\begin{array}{ccccc}
0 & I_{m} & 0 & \cdots & 0 \\
0 & 0 & I_{m} & \ddots & \vdots \\
\vdots & \vdots & \ddots & \ddots & 0 \\
0 & 0 & \cdots & 0 & I_{m} \\
-K_{1} & -K_{2} & \cdots & -K_{n} & -K_{n+1}
\end{array}\right]
$$

is negative semi-definite.

The input signal $u^{*}$ is not implementable, as $\mathbf{F}_{t}$ is unknown. Instead, an approximation of the ideal signal $u^{*}$ is generated as

$$
\widehat{\mathbf{u}}=\boldsymbol{\Phi}_{T}^{\mathrm{T}} \widehat{\boldsymbol{\Theta}}
$$

where $\widehat{\boldsymbol{\Theta}}$ is an approximation of $\boldsymbol{\Theta}^{*}$.

Then, replacing (3.12) in (3.6), yields

$$
\dot{\mathbf{Z}}_{n+1}=\mathbf{F}_{t}+\widehat{\mathbf{G}}_{u} \boldsymbol{\Phi}_{T}^{\mathrm{T}} \widehat{\boldsymbol{\Theta}}
$$

By adding and subtracting $\widehat{\mathbf{G}}_{u} \boldsymbol{\Phi}_{T}^{\mathrm{T}} \boldsymbol{\Theta}^{*}$ in (3.13), one can write

$$
\dot{\mathbf{Z}}_{n+1}=\mathbf{F}_{t}+\widehat{\mathbf{G}}_{u} \boldsymbol{\Phi}_{T}^{\mathrm{T}} \widehat{\boldsymbol{\Theta}}-\widehat{\mathbf{G}}_{u} \boldsymbol{\Phi}_{T}^{\mathrm{T}} \boldsymbol{\Theta}^{*}+\widehat{\mathbf{G}}_{u} \boldsymbol{\Phi}_{T}^{\mathrm{T}} \boldsymbol{\Theta}^{*}=\mathbf{F}_{t}+\widehat{\mathbf{G}}_{u} \boldsymbol{\Phi}_{T}^{\mathrm{T}} \widetilde{\boldsymbol{\Theta}}+\widehat{\mathbf{G}}_{u} \boldsymbol{\Phi}_{T}^{\mathrm{T}} \boldsymbol{\Theta}^{*}
$$

where

$$
\widetilde{\Theta}=\widehat{\Theta}-\Theta^{*}
$$

denotes the parameter estimation error. By substituting (3.10) into (3.14), one obtains

$$
\dot{\mathbf{Z}}_{n+1}=-\mathbf{K}_{1} \mathbf{Z}_{1}-\mathbf{K}_{2} \mathbf{Z}_{2}-\ldots-\mathbf{K}_{n+1} \mathbf{Z}_{n+1}+\widehat{\mathbf{G}}_{u} \boldsymbol{\Phi}_{T}^{\mathrm{T}} \widetilde{\mathbf{\Theta}}
$$

and

$$
\dot{\mathbf{Z}}=\mathbf{A}_{c l} \mathbf{Z}+\mathbf{B}_{c l} \Phi_{T}^{\mathrm{T}} \widetilde{\boldsymbol{\Theta}}
$$

where

$$
\mathbf{Z}=\left[\mathbf{Z}_{1}, \mathbf{Z}_{2}, \cdots, \mathbf{Z}_{n+1}\right]^{\mathrm{T}} \quad \mathbf{B}_{c l}=\left[\mathbf{0}, \mathbf{0}, \ldots, \widehat{\mathbf{G}}_{u}\right]^{\mathrm{T}}
$$

Remark 3. $\mathbf{A}_{c l}$ in (3.11) is a negative semi-definite matrix, so the positive definite symmetric matrix $\mathbf{P}$ can be found that satisfy the algebraic Lyapunov equation

$$
\mathbf{A}_{c l}^{\mathrm{T}} \mathbf{P}+\mathbf{P} \mathbf{A}_{c l}=-\mathbf{Q}
$$

for any positive definite symmetric matrix $\mathbf{Q}$.

Theorem. Consider constrained nonlinear system (3.6). By applying the control input $\mathbf{u}=\boldsymbol{\Phi}_{T}^{\mathrm{T}} \boldsymbol{\Theta}$ and adaptive law $\dot{\hat{\boldsymbol{\Theta}}}=-2 \boldsymbol{\Gamma}^{\mathrm{T}} \boldsymbol{\Phi}_{T} \mathbf{B}_{c l}^{\mathrm{T}} \mathbf{P Z}$, the closed loop stability and tracking performance are guaranteed. 
More precisely

$$
\mathbf{u}=\left[\begin{array}{c}
u_{1} \\
u_{2} \\
\vdots \\
u_{m}
\end{array}\right]=\left[\begin{array}{c}
\boldsymbol{\Phi}_{T 1}^{\mathrm{T}} \boldsymbol{\Theta}_{1} \\
\boldsymbol{\Phi}_{T 2}^{\mathrm{T}} \boldsymbol{\Theta}_{2} \\
\vdots \\
\boldsymbol{\Phi}_{T m}^{\mathrm{T}} \boldsymbol{\Theta}_{m}
\end{array}\right]=\left[\begin{array}{cccc}
\boldsymbol{\Phi}_{T 1}^{\mathrm{T}} & \mathbf{0} & \mathbf{0} & \mathbf{0} \\
\mathbf{0} & \boldsymbol{\Phi}_{T 2}^{\mathrm{T}} & \mathbf{0} & \mathbf{0} \\
\mathbf{0} & \ddots & \ddots & \mathbf{0} \\
\mathbf{0} & \mathbf{0} & \mathbf{0} & \boldsymbol{\Phi}_{T m}^{\mathrm{T}}
\end{array}\right]\left[\begin{array}{c}
\boldsymbol{\Theta}_{1} \\
\boldsymbol{\Theta}_{2} \\
\vdots \\
\boldsymbol{\Theta}_{m}
\end{array}\right]=\boldsymbol{\Phi}_{T}^{\mathrm{T}} \boldsymbol{\Theta}
$$

and

$$
u_{i}=\Phi_{i}^{\mathrm{T}} \theta_{p i} Z_{2 i}+\Phi_{i}^{\mathrm{T}} \theta_{I i} Z_{1 i}+\Phi_{i}^{\mathrm{T}} \theta_{D i} Z_{3 i}=\boldsymbol{\Phi}_{\mathbf{T i}}{ }^{\mathrm{T}} \mathbf{\Theta}_{i}
$$

in which

$$
\mathbf{\Phi}_{T i}=\left[\Phi_{i} Z_{1 i}, \Phi_{i} Z_{2 i}, \Phi_{i} Z_{3 i}\right]^{\mathrm{T}} \quad \boldsymbol{\Theta}_{i}=\left[\theta_{I i}, \theta_{p i}, \theta_{D i}\right]
$$

and

$$
\begin{aligned}
& k_{p i}=g_{p i}\left(e_{i}, \frac{d e_{i}}{d t}\right)=\Phi_{i}^{\mathrm{T}} \theta_{p i} \quad k_{I i}=g_{I i}\left(e_{i}, \frac{d e_{i}}{d t}\right)=\Phi_{i}^{\mathrm{T}} \theta_{I i} \\
& k_{D i}=g_{D i}\left(e_{i}, \frac{d e_{i}}{d t}\right)=\Phi_{i}^{\mathrm{T}} \theta_{D i}
\end{aligned}
$$

Remark 4. The nonlinear functions $g_{p i}(\cdot), g_{I i}(\cdot)$ and $g_{D i}(\cdot)$ may be obtained by a formulation as $H(\mathbf{x})$ in $(3.2)$.

Proof. Choose the Lyapunov function candidate

$$
V(\mathbf{Z}, \widetilde{\boldsymbol{\Theta}})=\mathbf{Z}^{\mathrm{T}} \mathbf{P Z}+\frac{1}{2} \widetilde{\boldsymbol{\Theta}}^{\mathrm{T}} \boldsymbol{\Gamma}^{-1} \widetilde{\boldsymbol{\Theta}} \quad \boldsymbol{\Gamma}>0
$$

with $\mathbf{P}>0$ and $\boldsymbol{\Gamma}>0$. The time derivative of $V$ is given by

$$
\dot{V}(\mathbf{Z}, \widetilde{\boldsymbol{\Theta}})=\dot{\mathbf{Z}}^{\mathrm{T}} \mathbf{P} \mathbf{Z}+\mathbf{Z}^{\mathrm{T}} \mathbf{P} \dot{\mathbf{Z}}+\frac{1}{2} \dot{\boldsymbol{\Theta}}^{\mathrm{T}} \boldsymbol{\Gamma}^{-1} \widetilde{\boldsymbol{\Theta}}+\frac{1}{2} \widetilde{\boldsymbol{\Theta}}^{\mathrm{T}} \boldsymbol{\Gamma}^{-1} \dot{\widetilde{\boldsymbol{\Theta}}}
$$

By replacing (3.17) into (3.25) and some manipulations, one can obtain

$$
\begin{aligned}
& \dot{V}(\mathbf{Z}, \widetilde{\boldsymbol{\Theta}})=\mathbf{Z}^{\mathrm{T}}\left(\mathbf{A}_{c l}^{\mathrm{T}} \mathbf{P}+\mathbf{P} \mathbf{A}_{c l}\right) \mathbf{Z}+2 \mathbf{Z}^{\mathrm{T}} \mathbf{P} \mathbf{B}_{c l} \boldsymbol{\Phi}_{T}^{\mathrm{T}} \widetilde{\boldsymbol{\Theta}}+\dot{\widetilde{\boldsymbol{\Theta}}}^{\mathrm{T}} \boldsymbol{\Gamma}^{-1} \widetilde{\boldsymbol{\Theta}} \\
& \quad=-\mathbf{Z}^{\mathrm{T}} \mathbf{Q} \mathbf{Z}+\left(2 \mathbf{Z}^{\mathrm{T}} \mathbf{P} \mathbf{B}_{c l} \boldsymbol{\Phi}_{T}^{\mathrm{T}}+\dot{\widetilde{\boldsymbol{\Theta}}}^{\mathrm{T}} \boldsymbol{\Gamma}^{-1}\right) \widetilde{\boldsymbol{\Theta}}
\end{aligned}
$$

Then, by adopting the adaptation law

$$
\dot{\widehat{\boldsymbol{\Theta}}}=-2 \boldsymbol{\Gamma}^{\mathrm{T}} \boldsymbol{\Phi}_{T} \mathbf{B}_{c l}^{\mathrm{T}} \mathbf{P} \mathbf{Z}
$$

one can conclude

$$
\dot{V}(\mathbf{Z}, \widetilde{\boldsymbol{\Theta}})=-\mathbf{Z}^{\mathrm{T}} \mathbf{Q} \mathbf{Z}<0
$$

Thus, Barbalat's Lemma (Sastry and Shankar, 1999; Åström and Wittenmark, 2013) ensures that the vector $\mathbf{Z}$ is asymptotically converged to zero. 


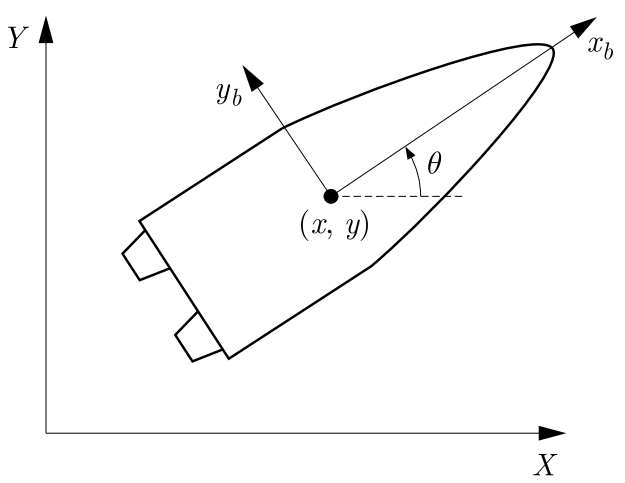

Fig. 2. Surface vessel in the inertial fixed and body fixed frames

\section{Simulation}

In this Section, the performance of the controller is evaluated in two situations, and the proposed method is applied to a surface vessel schematically shown in Fig. 2.

Such a three-input three-output system may be described by (Fang et al., 2004)

$$
\begin{aligned}
& m_{11} \dot{v}_{x}+d_{11} v_{x}=\tau_{1} \\
& m_{22} \dot{v}_{y}+m_{23} \dot{w}+d_{22} v_{v}+d_{23} w=\tau_{2} \\
& m_{33} \dot{w}+m_{23} \dot{v}_{y}+d_{23} v_{y}+d_{33} w=\tau_{3}
\end{aligned}
$$

in which $(x, y)$ and $\theta$ are respectively the surface vessel position and yaw angle in the inertial coordinate system and $\left(v_{x}, v_{y}\right)$, and $w$ denote respectively the surface vessel speed and rotational speed in the body coordinate system.

Dynamical equations (4.1) with using a set of simple mathematical operations can be rewritten in the form

$$
\mathbf{M}(\mathbf{q}) \dot{\mathbf{q}}+\mathbf{C}(\mathbf{q}, \dot{\mathbf{q}}) \dot{\mathbf{q}}+\mathbf{G}(\mathbf{q}, \dot{\mathbf{q}})=\tau^{*}
$$

where

$$
\begin{aligned}
& \mathbf{q}=[x, y, \theta]^{\mathrm{T}} \\
& \mathbf{M}(\mathbf{q})=\left[\begin{array}{ccc}
m_{11} \cos ^{2} \theta+m_{22} \sin ^{2} \theta & -m_{d} \cos \theta \sin \theta & -m_{23} \sin \theta \\
-m_{d} \cos \theta \sin \theta & m_{22} \cos ^{2} \theta+m_{11} \sin ^{2} \theta & m_{23} \cos \theta \\
-m_{23} \sin \theta & m_{23} \cos \theta & m_{33}
\end{array}\right] \\
& \mathbf{C}(\mathbf{q}, \dot{\mathbf{q}})=\left[\begin{array}{ccc}
\dot{\theta}\left(m_{d} \cos \theta \sin \theta\right) & \dot{\theta}\left(m_{11} \cos ^{2} \theta+m_{22} \sin ^{2} \theta\right) & 0 \\
-\dot{\theta}\left(m_{22} \cos ^{2} \theta+m_{11} \sin ^{2} \theta\right) & -\dot{\theta}\left(m_{d} \cos \theta \sin \theta\right) & 0 \\
-\dot{\theta}\left(m_{23} \cos \theta\right) & -\dot{\theta}\left(m_{23} \sin \theta\right) & 0
\end{array}\right]
\end{aligned}
$$

$$
\mathbf{G}(\mathbf{q}, \dot{\mathbf{q}})=\mathbf{K}(\mathbf{q}) \dot{\mathbf{q}}
$$$$
\mathbf{K}(\mathbf{q})=\left[\begin{array}{ccc}
d_{11} \cos ^{2} \theta+d_{22} \sin ^{2} \theta & -d_{d} \cos \theta \sin \theta & -d_{23} \sin \theta \\
-d_{d} \cos \theta \sin \theta & d_{22} \cos ^{2} \theta+d_{11} \sin ^{2} \theta & d_{23} \cos \theta \\
-d_{23} \sin \theta & d_{23} \cos \theta & d_{33}
\end{array}\right]
$$

and

$$
\boldsymbol{\tau}^{*}=\left[\tau_{1}, \tau_{2}, \tau_{3}\right]
$$

To facilitate the designing procedure, choose the state variables as

$$
\mathbf{X}_{1}=\mathbf{q} \quad \mathbf{X}_{2}=\dot{\mathbf{q}} \quad \mathbf{X}_{1}, \mathbf{X}_{2} \in \mathbb{R}^{3}
$$


The state space representation may be as

$$
\dot{\mathbf{X}}_{1}=\mathbf{X}_{2} \quad \dot{\mathbf{X}}_{2}=\mathbf{F}\left(\mathbf{X}_{1}, \mathbf{X}_{2}\right)+\mathbf{G}_{u}\left(\mathbf{X}_{1}, \mathbf{X}_{2}\right) \mathbf{u}
$$

where

$$
\mathbf{F}\left(\mathbf{X}_{1}, \mathbf{X}_{2}\right)=-\mathbf{M}^{-1}\left(\mathbf{X}_{1}\right)\left[\mathbf{C}\left(\mathbf{X}_{1}, \mathbf{X}_{2}\right) \mathbf{X}_{2}+\mathbf{G}\left(\mathbf{X}_{1}, \mathbf{X}_{2}\right)\right] \quad \mathbf{G}_{u}\left(\mathbf{X}_{1}, \mathbf{X}_{2}\right)=\mathbf{M}^{-1}\left(\mathbf{X}_{1}\right)
$$

and

$$
\mathbf{u}=\boldsymbol{\tau}^{*} \quad \mathbf{u} \in \mathbb{R}^{3}
$$

The numerical values of the model parameters in equation (4.1) are given in Table 1, as given by Fang et al. (2004).

Table 1. Model parameter values for the surface vessel

\begin{tabular}{|c|c|c|c|c|c|}
\hline Parameter & Value & Parameter & Value & Parameter & Value \\
\hline \hline$m_{11}[\mathrm{~kg}]$ & 1.0852 & $m_{33}[\mathrm{~kg}]$ & 0.2153 & $d_{11}[\mathrm{~kg} / \mathrm{s}]$ & 0.08656 \\
\hline$m_{22}[\mathrm{~kg}]$ & 2.0575 & $d_{11}[\mathrm{~kg}]$ & 0.08656 & $d_{22}[\mathrm{~kg} / \mathrm{s}]$ & 0.0762 \\
\hline$d_{33}[\mathrm{~kg} / \mathrm{s}]$ & 0.0031 & $d_{23}[\mathrm{~kg} / \mathrm{s}]$ & 0.151 & $d_{32}[\mathrm{~kg} / \mathrm{s}]$ & 0.0151 \\
\hline
\end{tabular}

The initial values and eigenvalues of the matrix $\mathbf{A}_{c l} \in \mathbb{R}^{9 \times 9}$ are selected as

$$
\begin{aligned}
& \mathbf{X}_{10}=[1,-1,0.3]^{\mathrm{T}} \quad \mathbf{X}_{20}=[0,0,0]^{\mathrm{T}} \\
& \boldsymbol{\lambda}=[-1,-1,-1,-1,-1,-1,-1,-1,-1]
\end{aligned}
$$

The matrix $\boldsymbol{\Gamma}$, constant scalars $\gamma_{i}, i=1,2,3$ and membership functions are chosen here as

$$
\boldsymbol{\Gamma}=\left[\begin{array}{ccc}
\gamma_{1} I_{L} & 0 & 0 \\
0 & \gamma_{2} I_{L} & 0 \\
0 & 0 & \gamma_{3} I_{L}
\end{array}\right] \quad \gamma_{1}=\gamma_{2}=10 \quad \gamma_{3}=10^{3}
$$

(a)

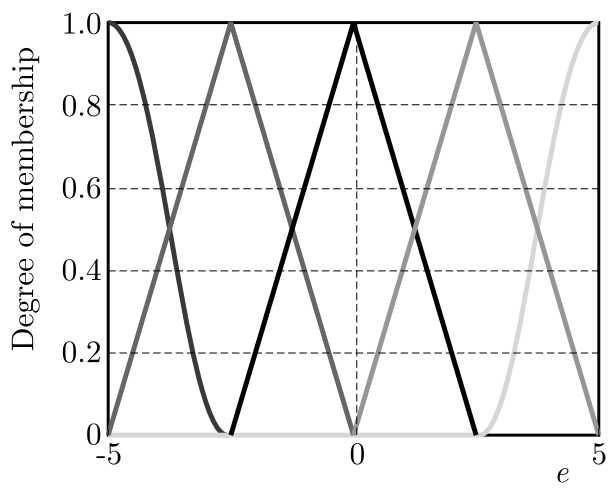

(b)

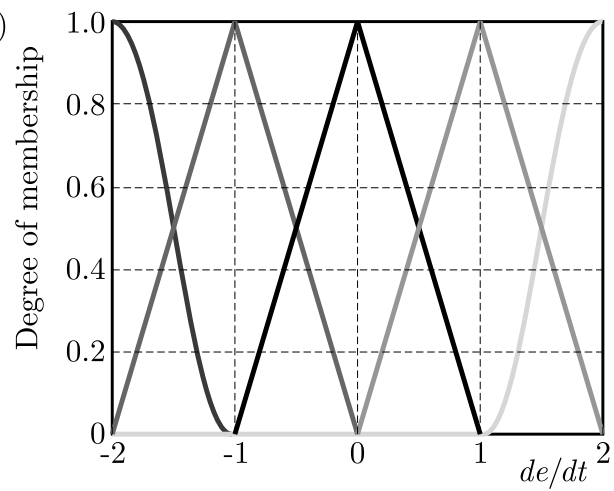

Fig. 3. (a) Membership function of the tracking error, (b) membership function of the derivative of the tracking error

Case $I$. The tracking performance of the proposed constrained control scheme is evaluated here and compared with that of the existing sliding mode method, see Zeinali and Leila (2010). Assume the reference position and the saturation limits are respectively given by $\left[x_{d}(t), y_{d}(t), \theta_{d}(t)\right]^{\mathrm{T}}=[3.5 \mathrm{~m}, 2 \mathrm{~m}, 0 \mathrm{rad}]^{\mathrm{T}}$ and $-2<\tau_{i}<2, i=1,2,3$. The external disturbance $\mathbf{d}(t)=(\sin (t)+1)[1,1,1]^{\mathrm{T}}$ also perturb the system at time $t=5 \mathrm{~s}$. 
Figures $4 \mathrm{a}$ and $4 \mathrm{~b}$ show that the tracking of the reference positions for $x$ and $y$ is obtained in the presence of disturbance. Figure 5 shows the capability of the proposed scheme in disturbance rejection compared with the sliding mode control by Zeinali and Notash (2010). The convergence of the controller coefficients $K_{p}, K_{D}, K_{I}$ for tracking $x_{d}(t), y_{d}(t)$ and $\theta_{d}(t)$ are demonstrated in Figs. 6 and 7. The control efforts in the proposed adaptive fuzzy method and the existing sliding controller are illustrated in Fig. 8.

(a)

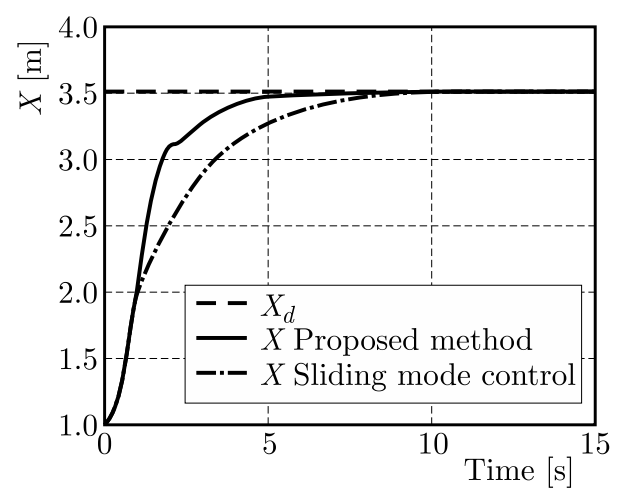

(b)

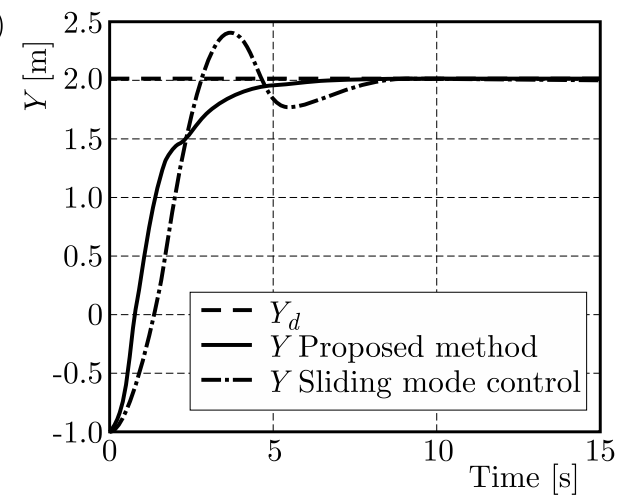

Fig. 4. (a) $X$ direction and (b) $Y$ direction tracking of the surface vessel

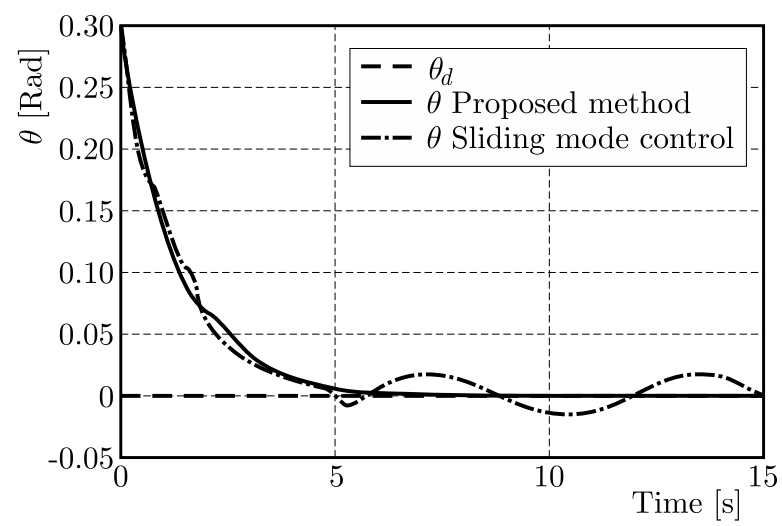

Fig. 5. Tracking of the pitch $\theta$ of the surface vessel

(a)
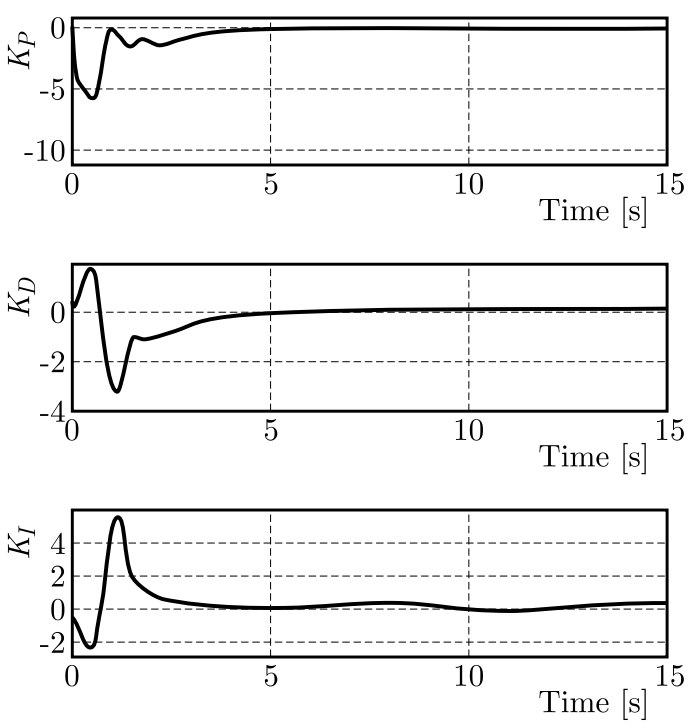

(b)
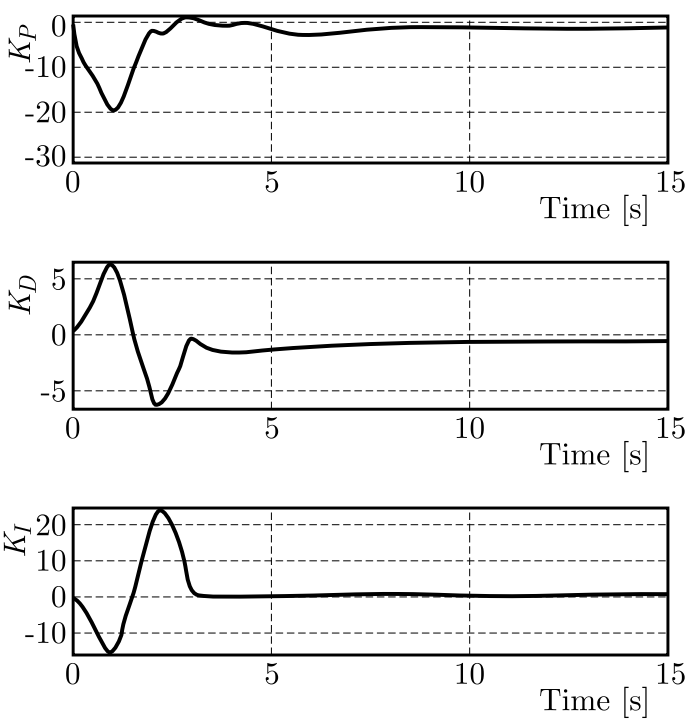

Fig. 6. Convergence of the controller parameters in (a) the $x$ direction, (b) the $y$ direction 

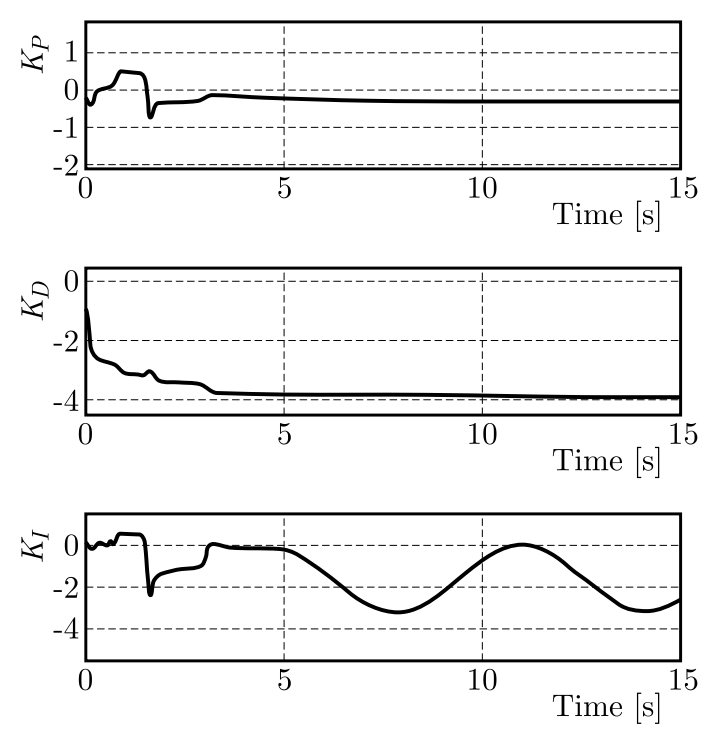

Fig. 7. Convergence of $K_{p}, K_{D}, K_{I}$ for the yaw controller
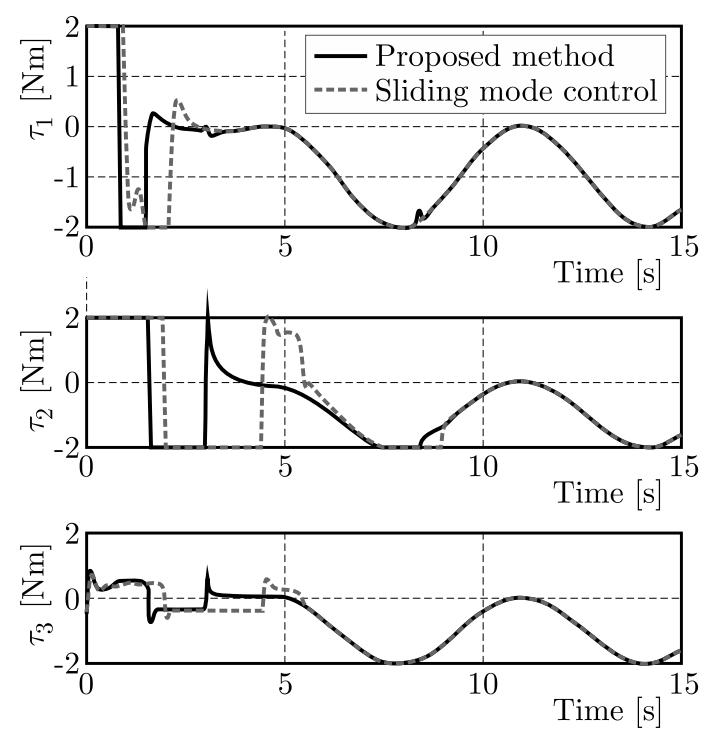

Fig. 8. Control signals in the proposed algorithm and the sliding control

To make a comparison between the designed adaptive fuzzy controller and the existing sliding control (Zeinali and Leila 2010), consider a cost function as

$$
J=\int_{0}^{t_{f}}\left(\|e(t)\|^{2}+\|u(t)\|^{2}\right) d t
$$

The lower cost of the proposed controller, as reported in Table 2, shows the advantage of the proposed approach.

Table 2. The costs of controllers in Case $I$

\begin{tabular}{|c|c|c|}
\hline Controller & Sliding mode & Proposed method \\
\hline \hline$J$ & 117.1378 & 61.3327 \\
\hline
\end{tabular}


Case $I I$. In this case, the reference signal and the saturation operator parameters are considered respectively as

$$
\left[\begin{array}{l}
x_{d}(t) \\
y_{d}(t) \\
\theta_{d}(t)
\end{array}\right]=\left[\begin{array}{c}
\sin (0.5 t) \\
\cos (0.5 t) \\
0
\end{array}\right] \quad \text { and } \quad-2<\tau_{i}<2 \quad i=1,2,3
$$

The simulation results, illustrated in Figs. 9 and 10, show that the proposed method gives smoother responses with less tracking error, compared with the sliding mode control (Zeinali and Leila, 2010). In the tracking of the reference output on the channel $y$, the sliding mode algorithm is unstable, while the proposed method is stable and the tracking error is converged to zero. Figure 11 shows that the control effort of the proposed method is much lower than that in the other method. Unlike the sliding mode, the control signal is zero in the steady state for the proposed method. Comparing the results may be also possible by adopting cost function (4.11), as numerically reported in Table 3.

(a)

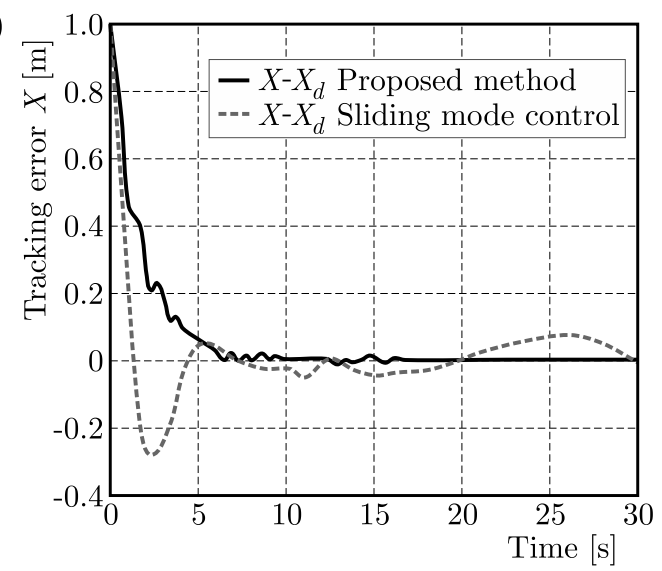

(b)

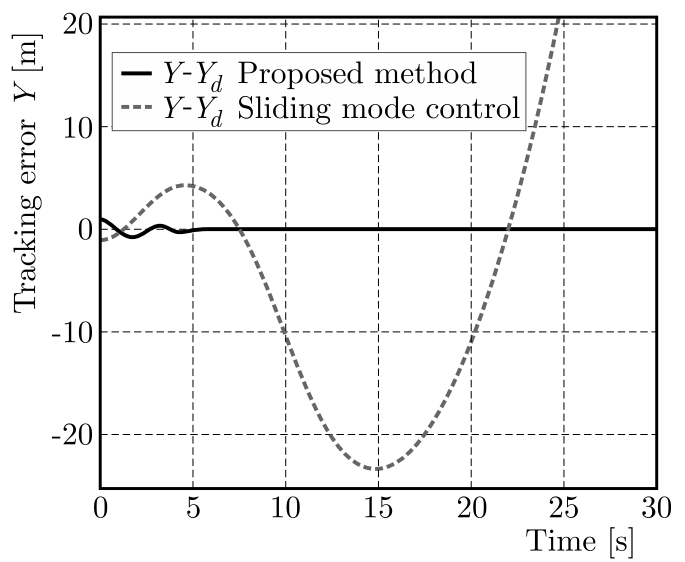

Fig. 9. Tracking error of the (a) the $x$ direction, (b) the $y$ direction

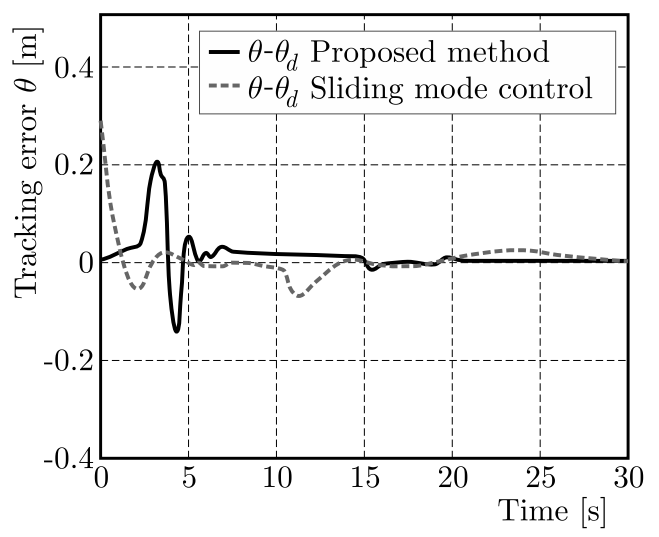

Fig. 10. Tracking error of pitch $\theta$

Table 3. The cost of controllers in Case $I I$

\begin{tabular}{|c|c|c|}
\hline Proposed method & Sliding mode & Controller \\
\hline \hline$J$ & 16933.4 & 77.8 \\
\hline
\end{tabular}



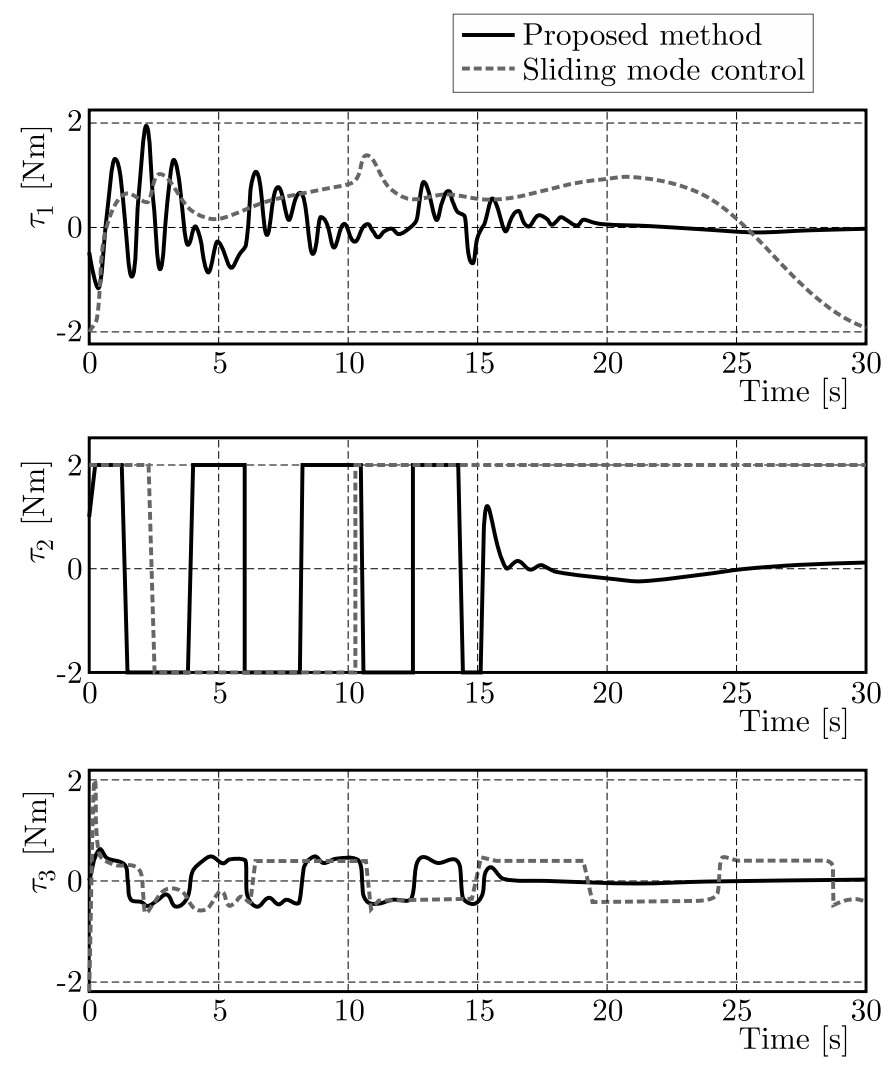

Fig. 11. Control signals

\section{Conclusion}

Focusing on the constraints on the inputs of nonlinear systems, the problem of robust tracking is investigated here. To solve the problem, an adaptive fuzzy algorithm is proposed for which the robust stability is proved using the Lyapunov stability theorem. As a practical situation, the problem is formulated for a surface vessel, taking the limitations on the control input into account. The designed controller is applied and the simulation results are presented to show the benefits of the method. The existing sliding control is also applied to the vessel and a cost function is defined to compare the results with the proposed scheme. In addition to demonstrations, a cost function is defined, and a numerical comparison is also made to show the benefits of the adaptive fuzzy algorithm.

\section{References}

1. Aström K.J., Wittenmark B., 2013, Adaptive control, Courier Corporation

2. Chen M., Ge S.S., How B.V.E., 2010, Robust adaptive neural network control for a class of uncertain MIMO nonlinear systems with input nonlinearities, Neural Networks, IEEE Transactions, 21, $5,796-812$

3. Chen M., Zhou Y., Guo W.W., 2014 Robust tracking control for uncertain MIMO nonlinear systems with input saturation using RWNNDO, Neurocomputing, 144, 436-447

4. Dai S.L., WAng M., Wang C., 2015, Neural learning control of marine surface vessels with guaranteed transient tracking performance, IEEE Transactions on Industrial Electronics, DOI 10.1109/TIE.2015.2504553 
5. Daly J.M., Tribou M.J., Waslander S.L., 2012, A nonlinear path following controller for an underactuated unmanned surface vessel, IEEE/RSJ International Conference of Intelligent Robots and Systems (IROS), 82-87

6. Fang Y., Zergeroglu E., De Queiroz M.S., Dawson D.M., 2004, Global output feedback control of dynamically positioned surface vessels: an adaptive control approach, Mechatronics, 14, 4, 341-356

7. Koofigar H.R., Amelian S., 2013, Robust adaptive vibration control for a general class of structures in the presence of time-varying uncertainties and disturbances, Journal of Theoretical and Applied Mechanics, 51, 3, 533-541

8. LEE H., 2011, Robust adaptive fuzzy control by backstepping for a class of MIMO nonlinear systems, Fuzzy Systems, IEEE Transactions, 19, 2, 265-275

9. Li G., Li W., Hildre H.P., Zhang H., 2015, Online learning control of surface vessels for fine trajectory tracking, Journal of Marine Science and Technology, in press, DOI 10.1007/s00773$-015-0347-9$

10. Li Y., Li T., Jing X., 2014, Indirect adaptive fuzzy control for input and output constrained nonlinear systems using a barrier Lyapunov function, International Journal of Adaptive Control and Signal Processing, 28, 2, 184-199

11. Li Y., Tong S., Li T., 2013, Direct adaptive fuzzy backstepping control of uncertain nonlinear systems in the presence of input saturation, Neural Computing and Applications, 23, 5, 1207-1216

12. LU L., YAO B., 2014, Online constrained optimization based adaptive robust control of a class of MIMO nonlinear systems with matched uncertainties and input/state constraints, Automatica, 50, $3,864-873$

13. Mclain R.B., Henson M.A., Pottmann M., 1999, Direct adaptive control of partially known nonlinear systems, IEEE Transactions on Neural Networks, 10, 3, 714-721

14. Montaseri G., Yazdanpanah M.J., 2012 Adaptive control of uncertain nonlinear systems using mixed backstepping and Lyapunov redesign techniques, Communications in Nonlinear Science and Numerical Simulation, 17, 8, 3367-3380

15. Muñoz D.A., Marquardt W., 2013, Robust control design of a class of nonlinear input-and state-constrained systems, Annual Reviews in Control, 37, 2, 232-245

16. Petersen I.R., Tempo R., 2014, Robust control of uncertain systems: classical results and recent developments, Automatica, 50, 5, 1315-1335

17. Sastry S., 1999, Nonlinear Systems: Analysis, Stability, and Control, 10, New York: Springer

18. Shaocheng T., Jiantao T., Tao W., 2000, Fuzzy adaptive control of multivariable nonlinear systems, Fuzzy Sets and Systems, 111, 2, 153-167

19. Wang D., Liu D., Li H., Ma H., 2014, Neural-network-based robust optimal control design for a class of uncertain nonlinear systems via adaptive dynamic programming, Information Sciences, 282, $167-179$

20. Wang H.Q., Chen B., Lin C., 2013a, Adaptive neural tracking control for a class of stochastic nonlinear systems with unknown dead-zone, International Journal of Innovative Computing, Information and Control, 9, 8, 3257-3269

21. Wang H., Chen B., Liu X., Liu K., Lin C., 2013b, Robust adaptive fuzzy tracking control for pure-feedback stochastic nonlinear systems with input constraints, Cybernetics, IEEE Transactions, 43, 6, 2093-2104

22. Wuxi S., Hongquan W., Furong L., Xiangyu W., 2013, Adaptive fuzzy control for a class of nonlinear systems with input constraint and unknown control direction, Control and Decision Conference (CCDC), 2013 25th Chinese. IEEE 
23. Xu D., HuAng J., 2010, Robust adaptive control of a class of nonlinear systems and its applications, Circuits and Systems I: Regular Papers, IEEE Transactions, 57, 3, 691-702

24. Zeinali M., Notash L., 2010, Adaptive sliding mode control with uncertainty estimator for robot manipulators, Mechanism and Machine Theory, 45, 1, 80-90

Manuscript received June 5, 2015; accepted for print January 13, 2016 